\title{
Disc oedema in association with Hunter's syndrome: ocular histopathological findings
}

\author{
MARILYN BECK' AND GILLIAN COLE² \\ From the 'Department of Ophthalmology, University Hospital of Wales, Heath, Cardiff CF4 4XW, and the \\ ${ }^{2}$ Department of Pathology, Welsh National School of Medicine, Heath Park, Cardiff CF4 4XN
}

SUMmary The histopathology of the posterior half of one eye and the optic nerves of a man with Hunter's syndrome, who was known to have disc oedema without raised intracranial pressure for 11 years of his life, is reported. The possible pathogenesis of the disc oedema is discussed; an important contributory factor was the deposition of abnormal mucopolysaccharides within the sclera. This caused gross thickening of the sclera with compression of the optic nerve at the intrascleral level.

Hunter's syndrome was first described in $1917^{\prime}$ and is one of the mucopolysaccharidoses. McKusick ${ }^{2}$ subdivided this group of inherited disorders of connective tissue into seven types. Hunter's syndrome is classified as type II MPS and may be further subdivided into type IIA (severe) and type IIB (mild) depending on the degree of intellectual impairment. This disease is usually inherited as an X-linked recessive trait.

Clinically this syndrome is characterised by short stature, a gargoyle facies, stiff joints, hepatosplenomegaly, and cardiac abnormalities. Variable degrees of mental subnormality occur, and progressive deafness is a consistent feature. In the mild form survival has been reported to the sixth decade, whereas in the severe form death usually occurs before 15 years of age. Ocular abnormalities described in this condition include exophthalmos, hypertelorism, papilloedema, and a pigmentary retinopathy, but the cornea is usually macroscopically clear.

This patient with Hunter's syndrome has been previously described, ${ }^{3}$ as he was known to have disc oedema for 11 years but yet retained good visual acuity.

\section{Case report}

This man, born 9 December 1947, was diagnosed as having Hunter's syndrome when he was aged 4 years because of his small stature, facial appearance, deafness, hepatosplenomegaly, and joint restriction. $\mathrm{He}$ was of normal intelligence, but he suffered from recurrent chest infections as a child and had his first Correspondence to Marilyn Beck, FRCS episodes of heart failure when he was aged 25 years. A cousin also had Hunter's syndrome and died of respiratory failure following dental surgery.

He was first seen by an ophthalmologist in 1971 . His visual acuities with a hypermetropic correction were $6 / 6$ right and $6 / 6$ left eyes. Fundal examination was recorded as showing normal peripheral fundi but 'bilateral disc blurring.'

In 1972 he was found to have gross bilateral disc swelling, normal retinal vessels, and normal peripheral fundi. Visual fields recorded on the Goldmann perimeter were full peripherally, but the blind spots were enlarged. Fluorography confirmed that this was true disc oedema. He was then seen at six-monthly intervals until March 1982 . The corrected acuities $6 / 6$ right and left were retained despite the fact that his disc appearances remained unchanged. The visual fields remained full peripherally, but there were increasing scotomata centrally due to the development of a pigmentary retinopathy which was first noted in 1980. The patient died of respiratory complications following hip surgery in September 1982.

POST-MORTEM EXAMINATION

The patient had the characteristic external appearance of Hunter's syndrome. There was generalised thickening of bone and collagenous structures, with particular involvement of the hip joints, mitral valve, larynx, and trachea with marked tracheal stenosis.

Brain and spinal cord. The brain was large and weighed $1700 \mathrm{~g}$. The dura covering brain and spinal cord was markedly thickened. Coronal sections did 


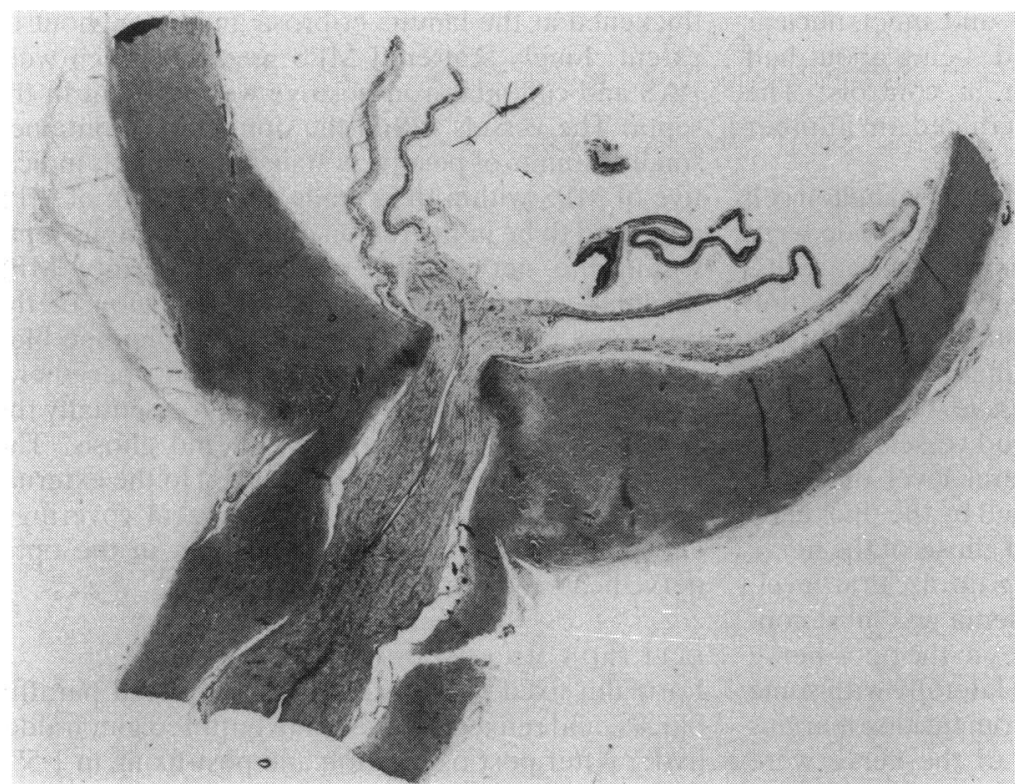

Fig. 1 Sections of posterior half of eye. The sclera is grossly thickened, and the intrascleral portion of the optic nerve is compressed. Bulging of the optic nerve is evident. ( $H E$, $\times 4 \cdot 7)$.

not show any macroscopic abnormality. The ventricles were of normal size. Microscopic examination showed the only obvious pathological change to be a mild deposition of mucopolysaccharides (MPS) within vessel walls, with a consequent perivascular rarefaction of tissue. No MPS accumulation within neurones or glial cells of the brain or cord was evident.

Eye and optic nerves. The posterior half of the right eye and both optic nerves were available for examination. Sections of the eye and optic nerves were embedded in paraffin and cut at $5 \mu \mathrm{m}$. Sections were

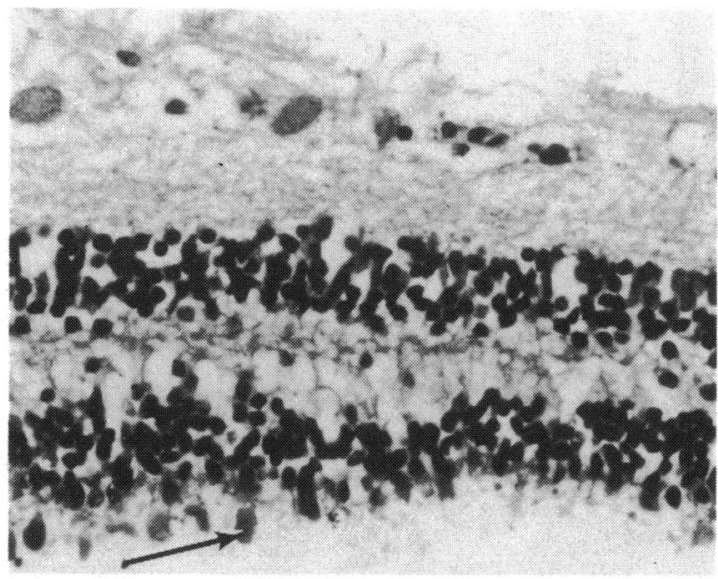

Fig. 2 Retina showing loss of rods and cones, and formation of amorphous structures (arrow). The outer and inner nuclear layers and the ganglion cells are reduced in number. $(H E, \times 250)$. stained with haematoxylin and eosin (HE), Kluver Barrer (KLB) stain for myelin, phosphotungstic acid haematoxylin (PTAH) for glial fibres and collagen, periodic acid schiff reagent (PAS) and colloidal iron for neutral and acid mucopolysaccharides.

MICROSCOPIC EXAMINATION

Sclera. The sclera was grossly thickened. Measurement of the thickness of the sclera at the posterior pole revealed it to be $2.9 \mathrm{~mm}$, which was nearly three times thicker than the average $1 \mathrm{~mm}$ thickness of a similar site in three control eyes (Fig. 1). The sclera was thickened by irregular bands of densely collagenous tissue. Scattered throughout the sclera were clumps of granular deposits, which were present in macrophages, and also apparently lying free in the tissue. The granular material proved to be MPS, staining positively with PAS and colloidal iron.

Choroid. The posterior choroid was the only uveal tissue available for examination and was essentially normal. There were sparsely scattered deposits of MPS confined to vessel walls. Bruch's membrane was intact.

Retina. Numerous levels of the posterior retina were examined. The retinal pigment layer had been completely destroyed, and there was marked loss of rods and cones. In those areas where rods and cones could still be detected the normal architecture was lost. Occasional eosinophilic amorphous structures were evident, and this was interpreted as a degenerative change (Fig. 2). The appearance of the rod and cone layer was not considered to be artefactual or due to poor fixation, since the other layers of the retina 
were well preserved. The outer and inner nuclear layers were considerably reduced, being about half the normal cell thickness seen in controls. The ganglion cells were likewise reduced in number (Fig. 2).

There was moderate gliosis within the ganglion cell layer. No deposits of acid mucopolysaccharide were evident within the retinal layers or ganglion cells. There was, however, a mild deposition of MPS within and around the vessel walls. Retinitis pigmentosa was present with accumulation of pigment within macrophages and lying free in the tissue. The pigment tended to accumulate around blood vessels.

Optic nerves. At the intrascleral level the optic nerve was considerably compressed by the thickened sclera (Fig. 1). There was marked gliosis of the nerve at the papilla (Fig. 3) and at the intrascleral level. Microscopic evidence of disc oedema was most convincingly demonstrated by changes in the optic nerve head, which bulged forwards and laterally with some displacement of the retina away from the disc margins (Fig. 1). The collagenous septa of the nerve were thickened at the lamina cribrosa and throughout its extent. Finely scattered MPS granules which were PAS and colloidal iron positive were present in the septa. The vessels within the optic nerve contained small amounts of positively staining material, indicative of MPS within their walls, but the bulk of MPS appeared to be in the region of the collagenous septa within the nerve. The presence of scanty MPS granules within macrophages in the region of the septa was best demonstrated on thin toluidine blue sections. Sections of the optic nerve in its peripheral extent from the optic chiasm revealed essentially the same features of thickened septa and gliosis. The gliosis, however, was mostly confined to the external fibres beneath the thickened meningeal coverings, and was less extensive than that seen in the optic nerve head and intrascleral portion.

ELECTRON MICROSCOPY

Formalin fixed tissue was rehydrated from paraffin blocks, and refixed in phosphate buffered glutaraldehyde. After post-osmication and post-fixing in 1.5\%

Fig. 3 Optic nerve papilla with marked glial fibre formation. (PTAH, ×250).






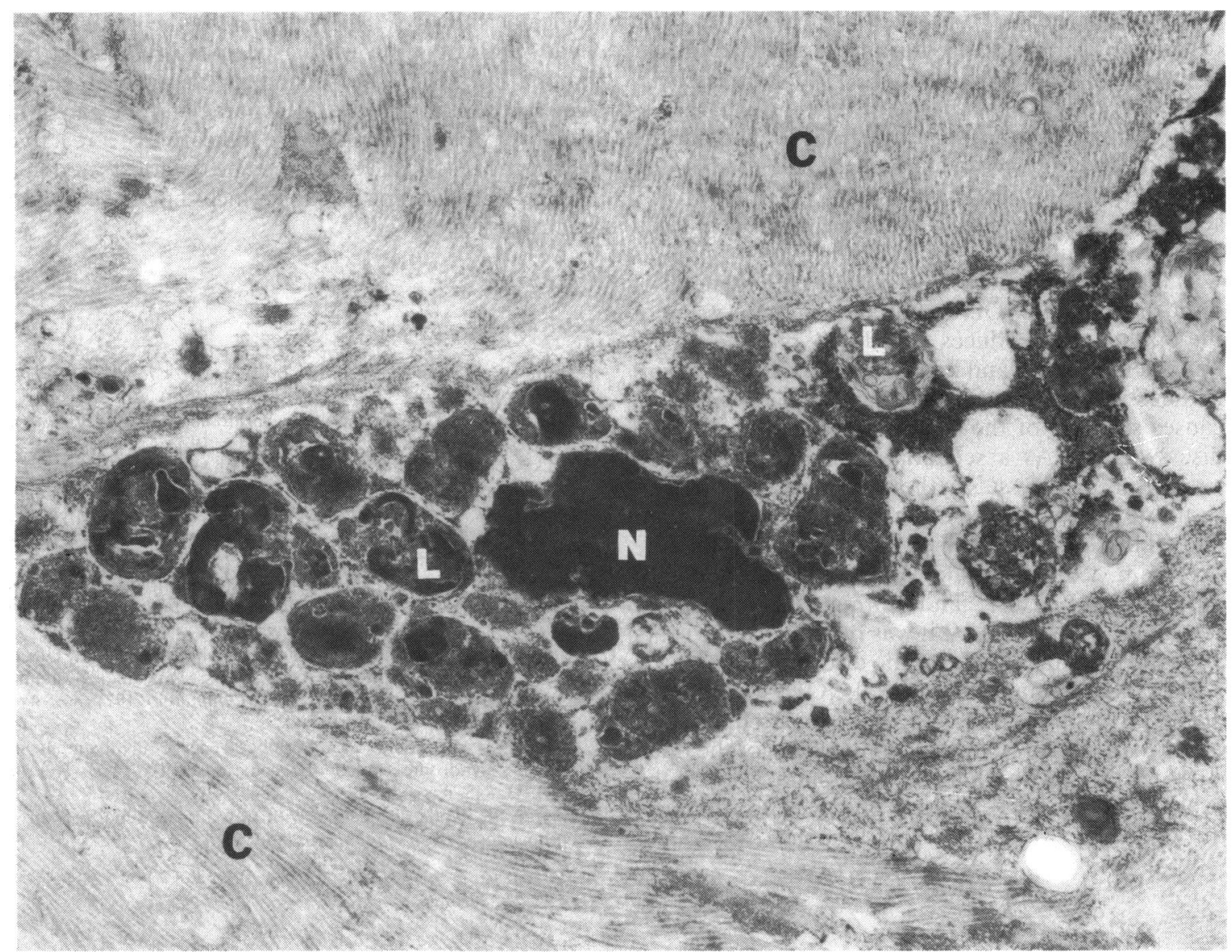

Fig. 4 Scleral fibroblast with intracytoplasmic membrane bound vacuoles containing lamellar and electron-dense inclusions. $N=$ nucleus. $L=$ lamellar inclusions. $("=$ collagen. $(\times 15000)$.

aqueous uranyl acetate, pieces of choroid, sclera, and optic nerve were dehydrated and embedded in Epon. Ultrathin sections were stained in lead citrate and viewed under a Philips 300 electron microscope.

In the choroid and sclera abnormal mucopolysaccharide accumulation was demonstrated by the presence of membrane bound vacuoles containing lamellar, granular, and electron-dense inclusions within the cytoplasm of fibroblasts (Fig. 4). Similar changes were evident in the optic nerve tissue, but the accumulation of MPS was better demonstrated in the ultrathin sections.

\section{Discussion}

Newell and Koistenen ${ }^{4}$ first discussed the pathological findings in eyes with systemic MPS. Later Goldberg and Duke ${ }^{5}$ and Topping et al. " reported the ocular histopathology and electron microscopy of the eyes of a patient with Hunter's syndrome. Disc oedema was not a feature in these reports, and the optic nerves were found to be normal. We have described the histopathology and ultrastructure of the posterior half of the eye of a patient with Hunter's syndrome who had unexplained disc oedema.

This case, similar to that reported by Goldberg and Duke, ${ }^{5}$ had retinal changes which were histologically indistinguishable from typical retinitis pigmentosa. Numerous cases of retinitis pigmentosa in association with MPS have been described, and it seems unlikely that this occurs as an independently inherited trait.

The accumulation of abnormal mucopolysaccharides within ocular structures was confirmed by electron microscopy. These changes are fully described by Topping et al."

During this patient's lifetime we speculated on the causes of the disc oedema. This was not thought to be due to elevated intracranial pressure as the cerebrospinal fluid (CSF) pressure was normal and CT scan showed no abnormality. 
Peripheral nerve infiltration with MPS has been described in Hunter's syndrome, ${ }^{7}$ and McKusick ${ }^{2}$ reported enlargement of the optic foramina in association with Hurler's syndrome. It was felt that gross infiltration of the optic nerves with MPS in this patient was unlikely to have been responsible for his disc oedema, as his optic nerve size was normal on CT scan and the optic foramina were not enlarged radiographically.

Hayreh $^{\times}$in his discussion on the pathogenesis of optic disc oedema (ODE) stated that compression of the optic nerve produces raised CSF pressure in the optic nerve sheath and an increase in tissue pressure in the nerve, thus causing axoplasmic flow stasis. This causes swelling of the optic nerve head and visible disc swelling. As a secondary change the swollen axons compress vessels in the prelaminar region, with resulting venous stasis. As the nerve impulse is conducted by the membrane of the axon and not by the axoplasm, axoplasmic flow stasis in itself does not impair visual acuity, and normal vision may be preserved. In our patient it was retained for 11 years after the first appearance of the disc oedema.

We consider that in this case the ODE was mainly due to gross thickening of the sclera by accumulation of MPS, producing external compression of the optic nerve together with some internal compression of the axons by the nerve septa which were similarly thickened by MPS. The disc oedema was histo- logically shown by the bulging of the optic nerve head, and the septa were especially involved at the lamina cribrosa. The secondary changes of gliosis were present within the optic nerve and the ganglion cell layer of the retina and are indicative of a reactive astrocytic response, which may be demonstrated in a variety of pathological changes including oedema and compression.

\section{References}

1 Hunter C. A rare disease in two brothers. Proc R Soc Med 1917; 10: 104-16.

2 McKusick VA. The mucopolysaccharidoses. Heritable disease of connective tissue 4th ed. St Louis: Mosby, 1972.

3 Beck M. Papilloedema in association with Hunter's syndrome. $\mathrm{Br}$ J Ophthalmol 1983; 67: 174-7.

4 Newell FW, Koistenen A. Lipochondrodystrophy (gargoylism): pathological findings in five cyes of threc patients. Arch Ophthalmol 1955; 53: 45-62.

5 Goldberg MF, Duke JR. Ocular histopathology in Hunter's syndrome. Systemic mucopolysaccharidoses type II. Arch Ophthalmol 1967; 77: 503-12.

6 Topping CM, Kenyon KR, Goldberg MF, Maumenec AE. Ultrastructural ocular pathology in Hunter's syndrome. Systemic mucopolysaccharidoses type II. Arch Ophthalmol 1971; 86: 16477.

7 Swift TR, McDonald DF. Peripheral nerve involvement in Hunter's syndrome (mucopolysaccharidoses II). Arch Neurol 1976; 33: 845-6.

8 Hayreh SS. Optic disc edema with raised intracranial pressure. Pathogenesis. Arch Ophthalmol 1977; 95: 1553-65. 